\title{
Short Sleep Duration and Physical and Psychological Health Outcomes Among Adult Survivors of Childhood Cancer
}

\author{
Margaret Lubas ${ }^{1}$, Belinda Mandrell ${ }^{2}$, Kirsten Ness $^{2}$, Kumar Srivastava ${ }^{3}$, Matthew \\ Ehrhardt ${ }^{1}$, Zhaoming Wang ${ }^{1}$, Melissa Hudson ${ }^{4}$, Leslie Robison ${ }^{2}$, Kevin Krull ${ }^{2}$, and Tara \\ Brinkman $^{2}$ \\ ${ }^{1}$ St Jude Children's Research Hospital \\ ${ }^{2}$ St. Jude Children's Research Hospital \\ ${ }^{3}$ St. Jude Children \\ ${ }^{4}$ St. Jude Children's Research Hospital
}

October 1, 2020

\begin{abstract}
Background: To examine associations between phenotypes of short sleep duration and clinically-assessed health conditions in long-term survivors of childhood cancer. Methods: Survivors recruited from the St. Jude Lifetime Cohort ( $\mathrm{n}=911 ; 52 \%$ female; mean age 34 years; 26 years post-diagnosis) completed behavioral health surveys and underwent comprehensive physical examinations. Sleep was assessed with the Pittsburgh Sleep Quality Index. Short sleep was defined as $<7$ hours per night with phenotypes of short sleep including poor sleep efficiency $(<85 \%)$, prolonged sleep onset latency ([?]30 minutes), and wake after sleep onset ([?]3 times per week). Covariates included childhood cancer treatment exposures, demographics, body mass index, and physical inactivity. Separate modified Poisson regression models were computed for each health category to estimate relative risks (RR) and 95\% confidence intervals (CI). Multinomial logistic regression models examined associations between sleep and an aggregated burden of chronic health conditions. Results: Short sleep duration was reported among $44 \%$ (95\% CI $41 \%-47 \%)$ of survivors. In multivariable models, short sleep duration alone was associated with pulmonary $(\mathrm{RR}=1.35,95 \%$ CI 1.08-1.69), endocrine ( $R R=1.22,95 \%$ CI 1.06-1.39) and gastrointestinal/hepatic conditions ( $R R=1.46,95 \%$ CI 1.18-1.79), and anxiety (RR 3.24, 95\% CI 1.64-6.41) and depression ( $\mathrm{RR}=2.33,95 \%$ CI 1.27-4.27). Short sleep with prolonged SOL was associated with a high/severe burden of health conditions $(\mathrm{OR}=2.35,95 \%$ CI 1.12-4.94). Conclusions: Short sleep duration was associated with multiple clinically-ascertained adverse health conditions. Although the temporality of these associations cannot be determined in this cross-sectional study, sleep is modifiable, and improving sleep may improve long-term health in survivors.
\end{abstract}

\section{Introduction}

Despite advances in cancer therapy and survival, childhood cancer survivors experience a lifelong risk for treatment-related late effects. ${ }^{1}$ Compared to the general population, long-term survivors have an increased risk of cardiovascular disease, ${ }^{2}$ metabolic syndrome, ${ }^{2}$ and obesity. ${ }^{2}$ Compared to sibling comparisons or noncancer controls, long-term survivors have a higher prevalence of cerebrovascular events, ${ }^{3}$ gastrointestinal complications ${ }^{4}$ and pulmonary deficits. ${ }^{5}$ Adult survivors of childhood cancer experience a high and variable burden of chronic health conditions ${ }^{6}$ increasing their risk of early mortality. ${ }^{7}$

While risk of adverse health outcomes in survivors is largely driven by treatment exposures, chronic health conditions can be exacerbated by poor health behaviors. Among a large cohort of adult survivors of childhood cancer, the observed association between unhealthy lifestyle behaviors (e.g. diet, physical activity) and metabolic syndrome was stronger than the association between cranial radiation and metabolic syndrome. ${ }^{8}$ 
Adherence to a Mediterranean diet has been associated with lower body mass index and lower risk of metabolic syndrome among leukemia survivors. ${ }^{9}$ Moreover, physical activity has been associated with decreased mortality risk among childhood cancer survivors. ${ }^{10}$ Identifying and promoting modifiable lifestyle behaviors may be essential to improving the health outcomes and quality of life of long-term survivors.

Associations between sleep and health have not been fully elucidated in survivors; however, in the general population sleep duration is widely associated with several chronic health conditions. In cross-sectional examinations of national samples, short and long sleep durations have been associated with metabolic syndrome,${ }^{11}$ cardiovascular disease,${ }^{12}$ and chronic kidney disease. ${ }^{13}$ Moreover, prospective associations between sleep disturbances and diabetes, ${ }^{14}$ depression, ${ }^{15}$ gastrointestinal disease ${ }^{16}$, and progression to end-stage renal disease ${ }^{17}$ have also been reported. The high prevalence of self-reported sleep disturbances among survivors of childhood cancer has been well characterized; ${ }^{18,19}$ however, few studies have examined health status and poor sleep among childhood cancer survivors. The purpose of this study was to examine associations between short sleep duration and clinically-assessed health conditions among adult survivors of childhood cancer.

\section{Methods}

\section{Study Participants}

Study participants were recruited from the St. Jude Lifetime Cohort Study (SJLIFE), ${ }^{20}$ a retrospective cohort of childhood cancer survivors who participate in prospective medical assessments. The cohort was initiated in 2007 and includes survivors of childhood cancer who are [?]5 years post-diagnosis of a pediatric cancer treated at St. Jude Children's Research Hospital. For the current study, survivors enrolled in SJLIFE who were [?]18 years of age and [?]10 years post diagnosis were recruited for a randomized controlled trial focused on sleep and cognition. We identified and screened 3,348 potentially eligible SJLIFE participants. Among these survivors, 562 refused participation in the intervention and 1,875 did not meet initial study specific inclusion/exclusion criteria (see Supplementary Table S1 intervention inclusion/exclusion criteria). This resulted in 911 participants who completed baseline evaluations to confirm eligibility for the intervention study and who form the study population for the current analysis. All participants provided written informed consent and the study was approved by the Institutional Review Board at St. Jude Children's Research Hospital.

\section{Measures}

\section{Health outcomes}

Physical health outcomes. Clinically-assessed health conditions were evaluated during on-campus medical evaluations and defined using modified Common Terminology Criteria for Adverse Events (CTCAE) version 4.03 criteria adapted for childhood cancer survivors. ${ }^{21}$ Comprehensive medical assessments included diagnostic procedures, an assessment of neuromuscular function, and a physical evaluation with a standardized laboratory battery. At the time of assessment, if survivors reported any chronic health conditions previously diagnosed, medical records were obtained to validate these diagnoses. The following organ systems were evaluated during the medical assessment and included as outcome variables: cardiac, pulmonary, renal, endocrine, gastrointestinal/hepatic, neurologic and musculoskeletal. CTCAE grades range from 1-4 (mild [grade 1], moderate [grade 2], severe or disabling [grade 3], life-threatening [grade 4]), and adverse health conditions were defined as grade [?]2 for each condition (i.e. moderate to life threatening). Supplemental Tables S2a-g present the frequency of grade 2-4 conditions for study participants, classified by each organ system. Chronic health conditions were included if they were diagnosed prior to and up to three months following the sleep assessment.

In addition to evaluating health conditions by each organ system, we created a composite measure assessing the burden/severity of the survivor's physical health. A method developed by Geenen et al. ${ }^{22}$ was employed to aggregate chronic health conditions across organ systems (cardiovascular, pulmonary, endocrine, neurologic, gastrointestinal, musculoskeletal, and renal), taking into account the frequency and grade of conditions. For 
survivors who had multiple chronic health conditions within the same organ system, the highest grade within that organ system was utilized. This chronic health burden score was classified via the following categories: none, low (only grade 1 events), medium (having [?]1 grade 2 event and/or 1 grade 3 event), high (having [?] 2 grade 3 events or 1 grade 4 event and at most 1 grade 3 event), and severe ( $>1$ grade 4 events or [?] 2 grade 3 events and 1 grade 4 event).

Psychological health outcomes. The Brief Symptom Inventory-18 (BSI-18) ${ }^{23}$ was administered at the time of the participant's sleep evaluation to assess clinically significant anxiety and depression. Using sex-specific normative data, survivors with a T-score [?]63 $\left(90^{\text {th }}\right.$ percentile) on either subscale were classified with clinically significant anxiety and/or depression. The BSI-18 has been previously validated in childhood cancer survivors. $^{24}$

\section{Self-reported sleep and short sleep duration (primary predictor).}

Phenotypes of self-reported short sleep were defined by utilizing questions from The Pittsburgh Sleep Quality Index (PSQI), which measures sleep quality and quantity over the previous month. Individual questions were used to operationalize phenotypes of habitual short sleep duration. Sleep duration was assessed via the question, "during the past month, how many hours of actual sleep did you get a night?" Short sleep was defined as self-reported sleep duration of $<7$ hours per night. Questions about sleep onset latency (SOL), wake after sleep onset (WASO), and sleep efficiency (SE) were also utilized to examine short sleepers with an additional sleep disturbance. Short sleepers with a prolonged SOL were defined as individuals who reported a SOL [?] 30 minutes. Short sleepers with a WASO impairment were defined as individuals who reported waking up late at night or early in the morning at least three times a week. Short sleepers with poor SE were defined by a SE $<85 \%$ which was calculated from the PSQI as the ratio of total sleep time compared to total time spent in bed. These classifications were not mutually exclusive and were defined as additional sleep disturbances among short sleepers.

\section{Covariates}

Treatment exposures (e.g. individual chemotherapeutic agents and radiation) known to increase the risk of chronic health conditions in survivors were selected a priori and informed by Children's Oncology Group guidelines version 5.0. ${ }^{26}$ Specific treatment exposures were selected for each health outcome in order to provide adequate adjustment for treatment exposures in separate models (see Table 1 for a description of treatment exposures included for each health outcome). Age at evaluation, age at cancer diagnosis, sex, race/ethnicity, body mass index (BMI) and physical inactivity were included as covariates in all models. Physical inactivity was defined as not meeting Centers for Disease Control recommendation for physical activity (75 minutes of vigorous or 150 minutes of moderate activity per week). ${ }^{27}$ Weekly moderate and vigorous activities were converted into metabolic equivalents (METS), individuals who reported $<450$ METS per week were classified as inactive. Age at evaluation, BMI and physical activity were assessed at the time of the sleep survey.

\section{Statistical analysis}

Descriptive statistics were calculated for all exposures, outcomes, and covariates. Differences between study variables by short sleep duration were reported by using Chi-square statistics for categorical variables and t-tests for continuous variables. Modified Poisson regression models with robust error variance ${ }^{28}$ were used to examine associations between phenotypes of short sleep duration and health outcomes. Separate models were conducted for each phenotype of short sleep and each health outcome, while adjusting for age at evaluation, sex, race/ethnicity, age at diagnosis, BMI, physical inactivity and condition-specific treatment exposures. When examining the associations between phenotypes of short sleep duration and the composite burden score of physical health outcomes, multinomial logistic regression was employed. Burden models were adjusted for age at evaluation, sex, race/ethnicity, BMI, and physical inactivity. All analyses were completed using SAS v9.4. All tests were two-sided and a p-value [?] .05 was considered statistically significant.

\section{Results}




\section{Self-Reported Sleep Characteristics}

Self-reported sleep characteristics of the study participants are shown in Table 2. On average participants reported a sleep duration of 6 hours and 38 minutes. Forty-four percent of the sample were classified as short sleepers, reporting a sleep duration of $<7$ hours per night. Only 14 participants reported a long sleep duration, defined as $>9$ hours per night. As previous research identifies a u-shaped relationship between sleep duration and adverse health outcomes ${ }^{12,13,29}$, the fourteen participants reporting long sleep duration were removed from subsequent analyses due to the inability to examine long sleepers separately.

\section{Demographic, Survivorship, and Health Characteristics}

Demographic, treatment exposures, and chronic health conditions among survivors with short and typical sleep duration are summarized in Table 3 . Survivors were $51.8 \%$ female, predominately non-Hispanic white $(84.1 \%)$, an average of 34 years of age, and 26 years from their cancer diagnosis. Leukemia (41.4\%), noncentral nervous system solid tumors $(23.4 \%)$, and Hodgkin lymphoma (13.2\%) were the most common diagnoses among participants. Grade 2 or higher cardiac (40.8\%), endocrine (50.0\%), neurologic (31.0\%), gastrointestinal/hepatic (31.1\%), musculoskeletal (30.1\%) and pulmonary $(26.3 \%)$ conditions were prevalent in the sample. Grade 2 or higher renal conditions (9.9\%), anxiety $(6.7 \%)$ and depression $(7.6 \%)$ were less common among survivors. Several health outcomes were more prevalent among short sleepers $(<7$ hours) compared to those with typical sleep duration ( 7 to 9 hours) such as: anxiety $10.6 \%$ vs. $3.4 \%(\mathrm{p}<0.0001)$, depression $10.3 \%$ vs. $5.2 \%$ ( $\mathrm{p}=0.0100)$, endocrine $55.8 \%$ vs. $45.5 \%(\mathrm{p}=0.0022)$, pulmonary $30.3 \%$ vs. $23.0 \%$ $(\mathrm{p}=0.0147)$, and gastrointestinal/hepatic conditions $37.0 \%$ vs. $26.3 \%(\mathrm{p}=0.0006)$ (Table 3$)$.

\section{Multivariable Analyses}

\section{Health Outcomes}

In multivariable models adjusted for condition-specific treatment exposures, demographic factors, BMI, and physical inactivity, all phenotypes of short sleep duration were associated with grade 2-4 pulmonary, endocrine, and gastrointestinal/hepatic conditions (Table 4). For example, short sleep duration alone was associated with a 22-46\% increased prevalence of pulmonary, (RR 1.35, 95\% CI 1.08-1.69), endocrine (RR 1.22, 95\% CI 1.06-1.39), and gastrointestinal/hepatic conditions (RR 1.46, 95\% CI 1.18-1.79) (Table 4). Short sleep with a prolonged SOL was associated with neurologic conditions (RR 1.32, 95\% CI 1.04-1.68) and short sleep with prolonged WASO with cardiac (RR 1.21, 95\% CI 1.02-1.42) and neurologic conditions (RR 1.25, 95\% CI 1.01-1.57) (Table 4). All phenotypes of short sleep duration were associated with anxiety (e.g. short sleep duration RR 3.24, 95\% CI 1.64-6.41) and depression (e.g. short sleep duration, RR 2.33, 95\% CI 1.27-4.27) (Table 4).

\section{Severity/Burden}

When aggregating physical health conditions to assess the burden of conditions, $34 \%$ of participants ( $\mathrm{n}=307$ ) were classified as having a high to severe burden score, $56 \%$ were classified as having a medium burden and $10 \%(n=90)$ were classified in the none to low reference group (Supplemental Table S3). In models adjusted for age, sex, race/ethnicity, BMI, and physical inactivity, short sleep duration with a prolonged SOL was associated with a high/severe burden of chronic health conditions (OR 2.35, 95\% CI 1.12-4.94) compared to those with a low to none burden. No other measures of short sleep duration were significantly associated with medium or high/severe burden of health conditions (Figure 1, Supplemental Table S4).

\section{Discussion}

In a large heterogeneous sample of adult survivors of childhood cancer, we found associations between phenotypes of short sleep duration and clinically assessed physical and psychological health outcomes. Short sleep duration with prolonged sleep onset latency increased risk of high/serve burden of chronic health conditions greater than 2-fold. Associations between all phenotypes of short sleep duration and psychological health outcomes ranged from 2 to 3 -fold in strength. While we did not observe large differences in the strength of associations between our phenotypes of short sleep duration and health outcomes, short sleep with 
prolonged SOL or WASO yielded consistent and strong associations to several health outcomes, including pulmonary, endocrine, gastrointestinal, neurologic, anxiety, and depression.

In our sample, $44 \%$ of survivors reported a habitual short sleep duration of less than seven hours per night. Among survivors with a short sleep duration, 21-27\% also reported a prolonged SOL and/or WASO, and nearly $30 \%$ of short sleepers met criteria for a poor sleep efficiency defined as $<85 \%$. Overall, $57 \%$ of the sample scored above the PSQI clinical cut-off for poor sleep quality. Consistent with previous literature, ${ }^{18,19}$ these findings underscore the severity and prevalence of sleep disturbances among survivors. Additionally, sleep disturbances ${ }^{30}$ and fatigue are associated with reduced quality of life ${ }^{19}$, neurocognitive impairment, ${ }^{30}$ and depression $^{19}$ among cross-sectional studies of childhood cancer survivors. One longitudinal study reported associations between dimensions of self-reported sleep and new onset psychological distress and new onset migraines. ${ }^{18}$ However, this study relied on self-reported health conditions. The current study utilized clinically-ascertained health conditions and highlights the range in which sleep duration relates to many biological systems (i.e. cardiac, pulmonary, gastrointestinal, and neurologic) and dimensions of psychological health.

There are several physiological pathways through which sleep duration could relate to adverse health conditions or overall poorer physical functioning. For example, sleep and immune functioning are bidirectionally related. Immune activation can alter sleep quality and patterns, while changes in sleep patterns can also impact the body's inflammatory response. ${ }^{31}$ Sleep patterns and duration are associated with the release of several hormones that regulate appetite, metabolism, and glucose tolerance. ${ }^{32,33}$ Other physiological processes follow diurnal patterns and may be disrupted by sleep disturbances. For instance, blood pressure often decreases at night (i.e. nocturnal dipping) and lack of decrease (i.e. non-dipping), which is associated with increased risk of cardiovascular disease, ${ }^{33}$ is more prevalent among individuals with sleep disturbances, such as obstructive sleep apnea and advanced sleep cycles. ${ }^{34}$ While there are several biological pathways through which sleep patterns and sleep disturbances could affect chronic health conditions, these relationships and their directions have not been fully elucidated. However, the potential causal significance of poor sleep and its associations with adverse health outcomes are reinforced by several prospective studies ${ }^{15-17} /$ reviews $^{14,29}$.

Understanding associations between sleep duration and chronic health conditions in childhood cancer survivors is important because sleep may serve as a modifiable point of intervention to improve the health of this vulnerable population. In survivorship, excess risk of chronic conditions is driven by treatment exposures; however, intervening on lifestyle factors can improve health outcomes in this population ${ }^{35}$. Several behavioral sleep interventions in non-cancer samples have been successful in improving sleep quantity and quality ${ }^{36,37}$ and some provide preliminary support for health improvements ${ }^{38-40}$ and thus, merit further study among cancer survivors.

The results of our study should be considered within the context of several limitations. Because our analyses were cross-sectional and sleep was measured at only one time point, we cannot assess the temporality of associations between sleep and health outcomes. In addition, chronic health conditions were assessed from the time of cohort participation up to three months after the sleep assessment. Although we acknowledge short sleep duration may have been chronic as well, we were unable to assess this in the current study. While there are several prospective studies identifying sleep preceding chronic health conditions, there is also strong potential for bidirectional associations. Moreover, as we examined a range of health conditions, it is possible that some chronic conditions could cause or contribute to sleep disturbances, while for others, sleep may be the antecedent. Despite our inability to ascertain the directionality of our found associations, sleep is modifiable and may serve as an important component of lifestyle interventions that aim to improve health among survivors. Interventions targeting sleep may be necessary irrespective of whether it is an antecedent or consequent of chronic health conditions.

The present study of long-term survivors of childhood cancer identifies associations between short sleep duration and several physical and psychological health conditions. While short sleep duration alone was consistently associated with several health conditions (e.g. pulmonary, endocrine, gastrointestinal/hepatic), short sleepers with prolonged SOL and/or WASO were associated with two additional health conditions 
(e.g., cardiac and neurologic conditions). Examining additional sleep disturbances among short sleepers may serve as a sensitive measure between sleep and health outcomes. In addition, SOL and/or WASO may severe as additional points of interventions when targeting sleep extension.

Conflict of interest statement: The authors have declared no conflicts of interest.

Acknowledgements: This work was supported by the National Cancer Institute at the National Institutes of Health (CA195547, M. Hudson and L. Robison, Principal Investigators; CA239689, T. Brinkman and K. Krull, Principal Investigators) and by the National Cancer Institute Training in Pediatric Cancer Care Survivorship award (5T32CA225590, K. Krull, Principal Investigator). The content is solely the responsibility of the authors and does not necessarily represent the official views of the National Institutes of Health. Support to St. Jude Children's Research Hospital was also provided by the Cancer Center Support (CORE) grant (CA21765, C. Roberts, Principal Investigator) and the American Lebanese Syrian Associated Charities (ALSAC).

Data sharing statement: The data that support the findings of this study are available from the corresponding author upon reasonable request

\section{References}

1. Hudson MM, Ness KK, Gurney JG, et al. Clinical ascertainment of health outcomes among adults treated for childhood cancer. JAMA.2013;309(22):2371-2381.

2. Nottage KA, Ness KK, Li C, Srivastava D, Robison LL, Hudson MM. Metabolic syndrome and cardiovascular risk among long-term survivors of acute lymphoblastic leukaemia - From the St. Jude Lifetime Cohort.Br J Haematol. 2014;165(3):364-374.

3. Bowers DC, McNeil DE, Liu Y, et al. Stroke as a late treatment effect of Hodgkin's Disease: a report from the Childhood Cancer Survivor Study.J Clin Oncol. 2005;23(27):6508-6515.

4. Goldsby R, Chen Y, Raber S, et al. Survivors of childhood cancer have increased risk of gastrointestinal complications later in life. Gastroenterology. 2011;140(5):1464-1471 e1461.

5. Armenian SH, Landier W, Francisco L, et al. Long-term pulmonary function in survivors of childhood cancer. J Clin Oncol.2015;33(14):1592-1600.

6. Bhakta N, Liu Q, Ness KK, et al. The cumulative burden of surviving childhood cancer: an initial report from the St Jude Lifetime Cohort Study (SJLIFE). Lancet. 2017;390(10112):2569-2582.

7. Bagnasco F, Caruso S, Andreano A, et al. Late mortality and causes of death among 5-year survivors of childhood cancer diagnosed in the period 1960-1999 and registered in the Italian Off-Therapy Registry. Eur $J$ Cancer. 2019;110:86-97.

8. Smith WA, Li C, Nottage KA, et al. Lifestyle and metabolic syndrome in adult survivors of childhood cancer: a report from the St. Jude Lifetime Cohort Study. Cancer. 2014;120(17):2742-2750.

9. Tonorezos ES, Robien K, Eshelman-Kent D, et al. Contribution of diet and physical activity to metabolic parameters among survivors of childhood leukemia. Cancer Causes Control. 2013;24(2):313-321.

10. Scott JM, Li N, Liu Q, et al. Association of Exercise With Mortality in Adult Survivors of Childhood Cancer. JAMA Oncol.2018;4(10):1352-1358.

11. Smiley A, King D, Bidulescu A. The Association between Sleep Duration and Metabolic Syndrome: The NHANES 2013/2014. Nutrients.2019;11(11).

12. Sabanayagam C, Shankar A. Sleep duration and cardiovascular disease: results from the National Health Interview Survey. Sleep.2010;33(8):1037-1042.

13. Salifu I, Tedla F, Pandey A, et al. Sleep duration and chronic kidney disease: analysis of the national health interview survey. Cardiorenal Med. 2014;4(3-4):210-216. 
14. Shan Z, Ma H, Xie M, et al. Sleep duration and risk of type 2 diabetes: a meta-analysis of prospective studies. Diabetes Care.2015;38(3):529-537.

15. Szklo-Coxe M, Young T, Peppard PE, Finn LA, Benca RM. Prospective associations of insomnia markers and symptoms with depression. Am J Epidemiol. 2010;171(6):709-720.

16. Ananthakrishnan AN, Long MD, Martin CF, Sandler RS, Kappelman MD. Sleep disturbance and risk of active disease in patients with Crohn's disease and ulcerative colitis. Clin Gastroenterol Hepatol.2013;11(8):965-971.

17. Yamamoto R, Shinzawa M, Isaka Y, et al. Sleep Quality and Sleep Duration with CKD are Associated with Progression to ESKD. Clin J Am Soc Nephrol. 2018;13(12):1825-1832.

18. Daniel LC, Wang M, Mulrooney DA, et al. Sleep, emotional distress, and physical health in survivors of childhood cancer: A report from the Childhood Cancer Survivor Study. Psychooncology.2019;28(4):903-912.

19. Mulrooney DA, Ness KK, Neglia JP, et al. Fatigue and sleep disturbance in adult survivors of childhood cancer: a report from the childhood cancer survivor study (CCSS). Sleep.2008;31(2):271-281.

20. Hudson MM, Ness KK, Nolan VG, et al. Prospective medical assessment of adults surviving childhood cancer: study design, cohort characteristics, and feasibility of the St. Jude Lifetime Cohort study.Pediatric blood \& cancer. 2011;56(5):825-836.

21. Hudson MM, Ehrhardt MJ, Bhakta N, et al. Approach for Classification and Severity Grading of Longterm and Late-Onset Health Events among Childhood Cancer Survivors in the St. Jude Lifetime Cohort. Cancer Epidemiol Biomarkers Prev. 2017;26(5):666-674.

22. Geenen MM, Cardous-Ubbink MC, Kremer LC, et al. Medical assessment of adverse health outcomes in long-term survivors of childhood cancer.JAMA. 2007;297(24):2705-2715.

23. Derogatis LR. Brief Symptom Inventory (BSI): Administration, scoring, and procedures manual. Minneapolis, MN: NCS Pearson; 2000.

24. Recklitis CJ, Parsons SK, Shih MC, Mertens A, Robison LL, Zeltzer L. Factor structure of the brief symptom inventory-18 in adult survivors of childhood cancer: results from the childhood cancer survivor study.Psychol Assess. 2006;18(1):22-32.

25. Buysse DJ, Reynolds CF, 3rd, Monk TH, Berman SR, Kupfer DJ. The Pittsburgh Sleep Quality Index: a new instrument for psychiatric practice and research. Psychiatry research. 1989;28(2):193-213.

26. Children's Oncology Group. Long-term follow-up guidelines for survivors of childhood, adolescent and young adult cancers, Version 5.0. Available from http://www.survivorshipguidelines.org/pdf/2018/COG_LTFU_Guidelines_v5.pdf. Published 2018. Accessed.

27. U.S. Department of Health and Human Services. Physical Activity Guidelines for Americans, 2nd edition. Washington, DC: U.S. : Department of Health and Human Services;2018.

28. Zou G. A modified poisson regression approach to prospective studies with binary data. Am J Epidemiol. 2004;159(7):702-706.

29. Cappuccio FP, D'Elia L, Strazzullo P, Miller MA. Sleep duration and all-cause mortality: a systematic review and meta-analysis of prospective studies. Sleep. 2010;33(5):585-592.

30. Tonning Olsson I, Lubas MM, Li C, et al. Insomnia and Neurocognitive Functioning in Adult Survivors of Childhood Cancer. JNCI Cancer Spectr. 2020;4(3):pkaa008.

31. Besedovsky L, Lange T, Haack M. The Sleep-Immune Crosstalk in Health and Disease. Physiol Rev. 2019;99(3):1325-1380. 
32. Leproult R, Van Cauter E. Role of sleep and sleep loss in hormonal release and metabolism. Endocr Dev. 2010;17:11-21.

33. Cappuccio FP, Miller MA. Sleep and Cardio-Metabolic Disease.Curr Cardiol Rep. 2017;19(11):110.

34. Thomas SJ, Booth JN, 3rd, Jaeger BC, et al. Association of Sleep Characteristics With Nocturnal Hypertension and Nondipping Blood Pressure in the CARDIA Study. J Am Heart Assoc.2020;9(7):e015062.

35. Golubic M, Schneeberger D, Kirkpatrick K, et al. Comprehensive Lifestyle Modification Intervention to Improve Chronic Disease Risk Factors and Quality of Life in Cancer Survivors. J Altern Complement Med. 2018;24(11):1085-1091.

36. Murawski B, Wade L, Plotnikoff RC, Lubans DR, Duncan MJ. A systematic review and meta-analysis of cognitive and behavioral interventions to improve sleep health in adults without sleep disorders.Sleep Med Rev. 2018;40:160-169.

37. Redeker NS, Caruso CC, Hashmi SD, Mullington JM, Grandner M, Morgenthaler TI. Workplace Interventions to Promote Sleep Health and an Alert, Healthy Workforce. J Clin Sleep Med. 2019;15(4):649-657.

38. Al Khatib HK, Hall WL, Creedon A, et al. Sleep extension is a feasible lifestyle intervention in freeliving adults who are habitually short sleepers: a potential strategy for decreasing intake of free sugars? A randomized controlled pilot study. Am J Clin Nutr.2018;107(1):43-53.

39. Baron KG, Duffecy J, Richardson D, Avery E, Rothschild S, Lane J. Technology Assisted Behavior Intervention to Extend Sleep Among Adults With Short Sleep Duration and Prehypertension/Stage 1 Hypertension: A Randomized Pilot Feasibility Study. J Clin Sleep Med.2019;15(11):1587-1597.

40. Chaput JP, Despres JP, Bouchard C, Tremblay A. Longer sleep duration associates with lower adiposity gain in adult short sleepers. Int J Obes (Lond). 2012;36(5):752-756.

[dataset] Brinkman TB, Lubas ML, Mandrell BN, Ness KK, Srivastava DK, Ehrhardt MJ, Wang Z, Hudson MM, Robison LL; 2020; MIND; available upon request.

Figures

Figure 1. Multivariable Associations between Phenotypes of Short Sleep and Severity/Burden of Physical Health Conditions

Figure Caption

Reference group = low burden/none; models adjusted for age, sex, race/ethnicity, body mass index, physical inactivity; long sleepers removed from analyses.

\section{Hosted file}

Table 1.pdf available at https://authorea.com/users/363398/articles/484206-short-sleepduration-and-physical-and-psychological-health-outcomes-among-adult-survivors-ofchildhood-cancer

\section{Hosted file}

Table 2.pdf available at https://authorea.com/users/363398/articles/484206-short-sleepduration-and-physical-and-psychological-health-outcomes-among-adult-survivors-ofchildhood-cancer

\section{Hosted file}

Table 3.pdf available at https://authorea.com/users/363398/articles/484206-short-sleepduration-and-physical-and-psychological-health-outcomes-among-adult-survivors-ofchildhood-cancer 


\section{Hosted file}

Table 4.pdf available at https://authorea.com/users/363398/articles/484206-short-sleepduration-and-physical-and-psychological-health-outcomes-among-adult-survivors-ofchildhood-cancer

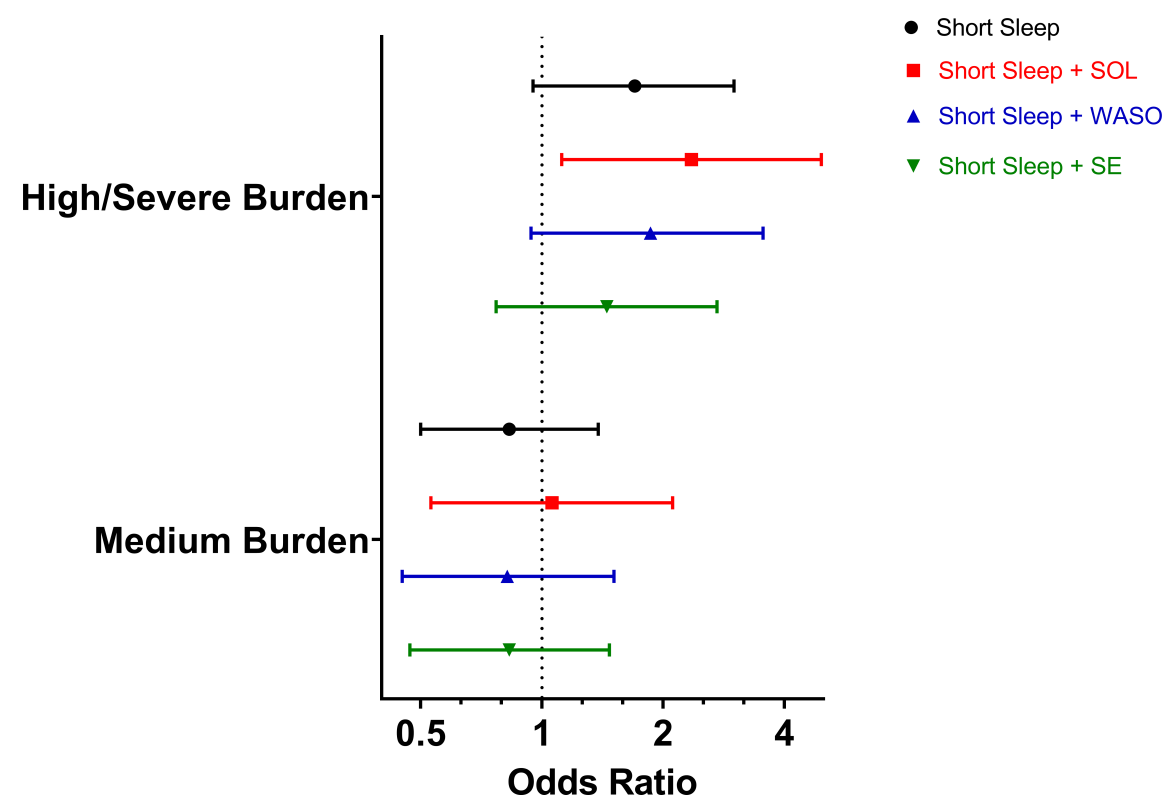

